\title{
Effects of Yoga on Sleep Quality, Depression, Anxiety, Stress, and Blood Glucose Levels Among Tertiary Students ${ }^{\dagger}$
}

\author{
Kokila Thiagarajah ${ }^{1, *}$, Natalia Shania Francis Peter ${ }^{1}$ \\ 1 Department of Allied Health Sciences, Faculty of Science, Universiti Tunku Abdul Rahman, Kampar, Perak - 31900, \\ Malaysia \\ * Correspondence: kokila@utar.edu.my; \\ $\uparrow$ Presented at International e-Conference on Bioengineering for Health and Environment (ICBHE 2020)
}

Received: 5.07.2020; Revised: 10.07.2020; Accepted: 12.07.2020; Published: 15.07.2020

\begin{abstract}
Sleep is defined as an easily reversible periodic state marked by the absence of wakefulness. Studies have shown that university students tend to have a diminished amount of sleep. This would lead to the loss of concentration, daytime sleepiness, and reduced academic performances. The aim of this study was to investigate the effects of yoga on overall sleep quality, depression, anxiety, stress, and blood glucose levels. A total of 88 participants with 44 students in each group, control and experimental, were recruited from Universiti Tunku Abdul Rahman based on the outcome of the Godin Shephard questionnaire. A total score of less than 14 was considered mild hence falling into the control group while above 14 and had practiced yoga for a minimum of 6 weeks were placed in the experimental group. The participants were required to self-administer a set of questionnaires consisting of sociodemographic information, the Pittsburgh Sleep Quality Index (PSQI) questionnaire and Depression, Anxiety, Stress Scores. Three components of sleep, namely the habitual sleep efficiency, sleep disturbances, and daytime dysfunctions, were better in the experimental group compared to the control one. Depression, anxiety, stress, and blood glucose levels also appeared to be more desirable in the experimental group with yoga students. It was found that blood glucose levels were positively correlated to the Global PSQI score, depression, anxiety, and stress measures in this study. In conclusion, yogapracticing students have healthier sleep patterns, negative emotional states, and blood glucose levels. In order to obtain more conclusive findings, similar studies should be carried out in different universities with larger sample size and for longer periods.
\end{abstract}

Keywords: Yoga; Blood glucose; Sleep; Stress; Students.

(C) 2020 by the authors. This article is an open-access article distributed under the terms and conditions of the Creative Commons Attribution (CC BY) license (https://creativecommons.org/licenses/by/4.0/).

\section{Funding}

This research received no external funding.

\section{Acknowledgments}

This study was approved for a year by UTAR scientific and ethical review committee and was supported by UTAR.

\section{Conflicts of Interest}

The authors declare no conflict of interest. 